\title{
Physico-Chemical Studies on Aqueous Solutions of (Amantadine and Oseltamivir) with PEG, Maltodextrin and SLS Binary Complex Formation
}

\author{
Subramanian Punitha ${ }^{1}$, Rajagpal Uvarani ${ }^{2}$ (D), Panneerselvam Arputham ${ }^{3}$ (i), \\ Subramanian Nithiyanantham 4 ,* (D)

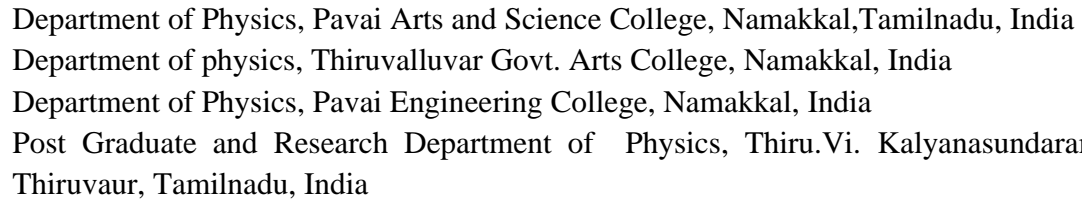

Received: 19.08.2020; Revised: 28.09.2020; Accepted: 30.09.2020; Published: 4.10.2020

\begin{abstract}
The molecular interaction between the drug and solvent molecules are discussed. The study aims to increase the solubility, stability, sweetness of drugs by way of complexation. The ultrasonic velocity, density, and viscosity have been measured at $2 \mathrm{MHz}$ for the aqueous solutions of (i) Influenza Anti-Viral Drugs + PEG, Maltodextrin, and SLS at different concentrations at a temperature 303K. Some derived parameters from the measured parameters have been computed. These properties are ascribing to molecular interactions through hydrogen bonding. The total absorption can be considered as the sum of contributions from solute-solvent interactions with FTIR studies.
\end{abstract}

Keywords: influenza anti-viral drugs; hydroxypropyl methyl cellulose; PEG; Maltodextrin; SLS and derived parameter.

(C) 2020 by the authors. This article is an open-access article distributed under the terms and conditions of the Creative
Commons Attribution (CC BY) license (https://creativecommons.org/licenses/by/4.0/).

\section{Introduction}

The importance of polymer blending has increased in recent years because the preparation of polymeric material with desired properties, low cost, and improved process stability complexation of different drugs with polymer, carbohydrate has been extensively studied in recent years with anti-viral drugs Nucleoside Analogue Reverse Transcriptase Inhibitors (NARTI) which also have analgesic properties. These phenomena can be minimized through the use of suitable drug carriers. In this sense, a current procedure is the inclusion of the complex formation of the drug with PEG [1-3]. Nevertheless, for various reasons, including cost, production capacity, and toxicology, the amount of dextrin that can be incorporated into drug formulation is limited [4]. Therefore, it is important to develop methods that can be applied to enhance the efficiency of the drug: PEG complexing [5] recently.

A mixture of polyethylene glycol with water are studied for their potential application in the biochemical and biomedical process [6]. Chemical and pharmaceutical applications include the PEG as a base material of ointments, as an anti-freezing agent, plasticizer, elementary substances for the production of cream and hydrogels. As a liberating agent for foam rubber, as a softener, antistatic agent, scouring agent, sizing agent, and dying auxiliary, 
it is used in many other cosmetic industries [7-10]. When certain polymers example, dextranpolyethylene glycol, are mixed with water above certain concentrations, the mixture separates into two immiscible aqueous phases. Each phase is rich preferentially in one of the polymers $[11,12]$. The nature and interactions of the components in the binary complex also change the drugs' chemical behavior $[13,14]$. Therefore, it is possible to expect that the structure of the guest also plays an important role in the binary. Influenza anti-viral drugs : polymer : surfactant complex formation and the effect that the complex has in the drugs' chemical and biological behavior.

Inclusion complexation with PEG is widely exploited to improve various drug molecules' solubility and stability [15]. Amantadine and Oseltamivir is a potent reverse transcriptase inhibitor of the class of Nucleoside Analogue Reverse Transcriptase Inhibitors (NARTI) [16]. On the other hand, for a series of reasons including cost, production capacity, possible toxicity, formulation bulk problems, pharmaceutical dosage forms should contain small amounts of possible polymers, dextrins, and surfactants. When polymers are added, all the data are noticeable to show that the polymer's influence on the complexing ability of dextrin depends on the nature of the dextrin and the polymer interactions between both of them. The nature and interactions of the components in the binary complex also change the drug's chemical behavior. The addition of a third component, such as water-soluble polymers, enhances the efficiency of drug-cyclodextrin complexation. This solubilization enhancement is synergistic [17-19].

\section{Materials and Methods}

\subsection{Materials and methodology.}

The pure samples of these polymers and carbohydrates are obtained from Madras scientific Chemicals, Salem. The drug gift samples are obtained from Sun plasma, Mumbai, India. (i)Initially, the binary mixtures of anti-influenza viral drugs (1\%) + PEG, Maltodextrin, SLS aqueous solutions are prepared. The ultrasonic velocity and absorption studies are undertaken in the aqueous solutions of anti-influenza viral drugs (1\%) + PEG, Maltodextrin, SLS, to understand the interaction's nature between the two different solutes at $303 \mathrm{~K}$. The ultrasonic velocities of solutions were measured using a single frequency continuous wave ultrasonic interferometer (Model F81, Mittal Enterprises) with an accuracy of $\pm 0.05 \%$ of $2 \mathrm{MHz}$ at $303 \mathrm{~K}$ maintained with a constant temperature bath. The densities of the solutions were measured using a specific gravity bottle with an accuracy of $\pm 0.01 \mathrm{kgm}^{-3}$. The viscosity was measured using Ostwald's viscometer to an accuracy of $\pm 0.2 \%$. The FTIR spectra were collected for these samples using Fourier Transform Infra-Red Spectrometer. Model: Spectrum RX, Perkin Elmer.

\subsection{Physical parameters.}

Acoustical and thermodynamical parameters were calculated from the empirical Jacobson's relations [20].

$$
\text { Adiabatic compressibility } \quad \beta=1 / \mathrm{u}^{2} \rho
$$

has been calculated from the u-ultrasonic velocity and $\rho$ - density values of the components using the Newton -Laplace equation.

$$
\text { Intermolecular free length } \quad \mathrm{L}_{\mathrm{f}}=\mathrm{K}_{\mathrm{T}} \beta^{1 / 2}
$$


Where $\mathrm{K}_{\mathrm{T}}$ is the temperature-dependent constant known as Jacobson's constant $\left(\mathrm{K}_{\mathrm{T}}=2.131 \times 10^{-6}\right)$, and $\beta$ is the adiabatic compressibility

$$
\text { Internal pressure } \quad \pi_{\mathrm{i}}=\mathrm{bRT}[\mathrm{K} \eta / \mathrm{u}]^{1 / 2} \rho^{2 / 3} / \mathrm{M}^{7 / 6}
$$

Where, $\mathrm{b}$ stands for cubic packing, which is assumed to be 2 for all liquids, T-absolute temperature in Kelvin, Where $M_{\text {eff }}$ is the effective molecular weight of the mixture $\left(M_{\text {eff }}=\sum m_{i}\right.$ $\mathrm{x}_{\mathrm{i}}$,Where $\mathrm{m}_{\mathrm{i}}$ and $\mathrm{x}_{\mathrm{i}}$ are the molecular weight and mole fraction of individual constituents, respectively $\mathrm{K}$ is an independent temperature constant which is equal to $4.281 \times 10^{9}$ [21] for all liquids, $\mathrm{R}$ is the universal gas constant, $\eta$-Viscosity of the solution).

Rao's constant

Relaxation time

Acoustic impedance

Absorption coefficient

Free Volume

Cohesive energy

Solvation number

$$
\begin{gathered}
\mathrm{R}_{\mathrm{a}}=(\mathrm{M} / \rho)(\mathrm{u})^{1 / 3} \\
\tau=4 / 3 \beta \eta \\
\mathrm{Za}=\rho \mathrm{u} \\
\alpha / \mathrm{f}^{2}=\left(8 \pi^{2} \eta / 3 \rho u^{2}\right) \\
\mathrm{V}_{\mathrm{f}}=\left(\mathrm{M}_{\mathrm{eff}} \mathrm{u} / \mathrm{K} \eta\right)^{3 / 2} \\
\mathrm{CE}=\mathrm{V}_{\mathrm{f}} \pi_{\mathrm{i}} \\
\mathrm{Sn}=\mathrm{M}_{2} / \mathrm{M}_{1}[1-((\beta) / \beta \mathrm{o})][(100-\mathrm{x}) / \mathrm{x}]
\end{gathered}
$$

Where $M_{1}, M_{2}$ are the molecular weight of the solvent and solute, and $\beta_{o}$ are the adiabatic compressibility of solution and solvent.

\section{Results and Discussion}

Using the measured values and their respective derived parameters' are presented in Table 1.

Table 1 shows that velocity, density, and viscosity gradually increase with the increase in concentration. In Influenza anti-viral drugs (1\%) + PEG, Maltodextrin, and SLS, it is observed that the adiabatic compressibility, intermolecular free length, internal pressure, absorption co-efficient, cohesive energy, and relaxation time decreases with an increase in the concentration of a solution of all the systems. Further, Rao's constant, free volume, acoustical

\begin{tabular}{|c|c|c|c|c|c|c|}
\hline $\begin{array}{c}\text { Conc. } \\
\%\end{array}$ & $\underset{\mathbf{m s}^{-1}}{\mathbf{U}}$ & $\underset{\mathrm{kgm}^{-3}}{\rho}$ & $\begin{array}{c}\eta \\
\mathbf{x 1 0 ^ { - 3 }} \\
\mathbf{N s m}^{-2}\end{array}$ & $\begin{array}{c}\beta \\
\mathbf{x 1 0}^{-10} \\
\mathbf{N}^{-1} \mathbf{m}^{2}\end{array}$ & $\begin{array}{l}\mathbf{L}_{\mathbf{f}} \\
\AA\end{array}$ & $\begin{array}{c}\pi_{\mathrm{i}} \\
\mathbf{x 1 0}^{6} \\
\text { Pascal }\end{array}$ \\
\hline \multicolumn{7}{|c|}{ Amantadine + PEG } \\
\hline 0.00 & 1531 & 1010 & 0.912 & 4.224 & 0.410 & 0.875 \\
\hline 0.25 & 1535 & 1048 & 0.945 & 4.049 & 0.401 & 0.352 \\
\hline 0.50 & 1540 & 1061 & 0.958 & 3.974 & 0.397 & 0.210 \\
\hline 0.75 & 1546 & 1070 & 0.971 & 3.910 & 0.394 & 0.147 \\
\hline 1.00 & 1551 & 1082 & 0.983 & 3.841 & 0.391 & 0.112 \\
\hline 1.25 & 1557 & 1091 & 0.999 & 3.780 & 0.387 & 0.090 \\
\hline 1.50 & 1561 & 1099 & 1.095 & 3.734 & 0.385 & 0.078 \\
\hline 1.75 & 1565 & 1111 & 1.124 & 3.675 & 0.382 & 0.067 \\
\hline 2.00 & 1569 & 1122 & 1.141 & 3.620 & 0.379 & 0.059 \\
\hline \multicolumn{7}{|c|}{ Oseltamivir + PEG } \\
\hline 0.00 & 1525 & 1007 & 0.894 & 4.270 & 0.412 & 0.867 \\
\hline 0.25 & 1522 & 1024 & 0.910 & 4.215 & 0.409 & 0.342 \\
\hline 0.50 & 1528 & 1031 & 0.926 & 4.154 & 0.406 & 0.204 \\
\hline 0.75 & 1535 & 1038 & 0.938 & 4.088 & 0.403 & 0.142 \\
\hline 1.00 & 1542 & 1044 & 0.947 & 4.028 & 0.400 & 0.108 \\
\hline 1.25 & 1548 & 1052 & 0.959 & 3.966 & 0.397 & 0.087 \\
\hline
\end{tabular}
impedance, and solvation number increases with the increase in concentration can also be observed. Their chemical structures are shown in Fig.1.

Table 1. Ultrasonic velocity $(\mathrm{U})$, density $(\rho)$, viscosity $(\eta)$, adiabatic compressibility $(\beta)$ free length $\left(\mathrm{L}_{\mathrm{f}}\right)$, internal pressure $\left(\pi_{i}\right)$, molar sound velocity $(\mathrm{R})$, absorption cefficient $\left(\alpha / \mathrm{f}^{2}\right)$ free volume $\left(\mathrm{V}_{\mathrm{f}}\right)$, cohesive energy (CE ), relaxation time $(\tau)$ acoustical impedance $\left(Z_{a}\right)$ and salvation number $\left(S_{n}\right)$ for (i) Amantadine + PEG, (ii) Oseltamivir + PEG, (iii) Amantadine + Maltodextrin, (iv) Oseltamivir + Maltodextrin , (v) Amantadine + SLS and (vi) Oseltamivir + SLS in aqueous solution at 303K 


\begin{tabular}{|c|c|c|c|c|c|c|}
\hline 1.50 & 1555 & 1059 & 0.967 & 3.905 & 0.394 & 0.072 \\
\hline 1.75 & 1561 & 1066 & 0.974 & 3.849 & 0.391 & 0.061 \\
\hline 2.00 & 1567 & 1074 & 0.986 & 3.791 & 0.385 & 0.053 \\
\hline \multicolumn{7}{|c|}{ Amantadine + Maltodextrin } \\
\hline 0.00 & 1531 & 1010 & 0.912 & 4.224 & 0.410 & 0.545 \\
\hline 0.25 & 1533 & 1016 & 0.923 & 4.188 & 0.408 & 0.256 \\
\hline 0.50 & 1536 & 1022 & 0.937 & 4.147 & 0.406 & 0.163 \\
\hline 0.75 & 1539 & 1028 & 0.956 & 4.107 & 0.404 & 0.119 \\
\hline 1.00 & 1543 & 1036 & 0.975 & 4.054 & 0.401 & 0.093 \\
\hline 1.25 & 1547 & 1043 & 0.996 & 4.006 & 0.399 & 0.077 \\
\hline 1.50 & 1551 & 1050 & 1.058 & 3.959 & 0.397 & 0.066 \\
\hline 1.75 & 1555 & 1057 & 1.094 & 3.912 & 0.394 & 0.058 \\
\hline 2.00 & 1559 & 1064 & 1.123 & 3.866 & 0.392 & 0.051 \\
\hline \multicolumn{7}{|c|}{ Oseltamivir + Maltodextrin } \\
\hline 0.00 & 1525 & 1007 & 0.894 & 4.270 & 0.412 & 0.540 \\
\hline 0.25 & 1529 & 1028 & 0.905 & 4.160 & 0.407 & 0.256 \\
\hline 0.50 & 1535 & 1036 & 0.912 & 4.096 & 0.403 & 0.163 \\
\hline 0.75 & 1541 & 1043 & 0.924 & 4.037 & 0.400 & 0.118 \\
\hline 1.00 & 1546 & 1052 & 0.936 & 3.977 & 0.397 & 0.092 \\
\hline 1.25 & 1551 & 1061 & 0.944 & 3.917 & 0.394 & 0.075 \\
\hline 1.50 & 1556 & 1069 & 0.956 & 3.863 & 0.392 & 0.064 \\
\hline 1.75 & 1562 & 1077 & 0.967 & 3.805 & 0.389 & 0.055 \\
\hline 2.00 & 1567 & 1083 & 0.974 & 3.760 & 0.386 & 0.048 \\
\hline \multicolumn{7}{|c|}{ Amantadine + SLS } \\
\hline 0.00 & 1531 & 1010 & 0.912 & 4.224 & 0.410 & 0.871 \\
\hline 0.25 & 1535 & 1027 & 0.934 & 4.132 & 0.405 & 0.338 \\
\hline 0.50 & 1539 & 1034 & 0.949 & 4.083 & 0.403 & 0.202 \\
\hline 0.75 & 1543 & 1042 & 0.963 & 4.030 & 0.400 & 0.141 \\
\hline 1.00 & 1546 & 1053 & 0.979 & 3.973 & 0.397 & 0.108 \\
\hline 1.25 & 1551 & 1061 & 0.994 & 3.917 & 0.394 & 0.087 \\
\hline 1.50 & 1555 & 1069 & 1.031 & 3.868 & 0.392 & 0.074 \\
\hline 1.75 & 1559 & 1074 & 1.064 & 3.830 & 0.390 & 0.064 \\
\hline 2.00 & 1562 & 1082 & 1.132 & 3.788 & 0.388 & 0.057 \\
\hline \multicolumn{7}{|c|}{ Oseltamivir + SLS } \\
\hline 0.00 & 1525 & 1007 & 0.894 & 4.270 & 0.412 & 0.862 \\
\hline 0.25 & 1531 & 1023 & 0.921 & 4.170 & 0.407 & 0.335 \\
\hline 0.50 & 1535 & 1030 & 0.936 & 4.120 & 0.405 & 0.200 \\
\hline 0.75 & 1539 & 1038 & 0.945 & 4.067 & 0.402 & 0.140 \\
\hline 1.00 & 1543 & 1044 & 0.958 & 4.023 & 0.400 & 0.107 \\
\hline 1.25 & 1547 & 1051 & 0.969 & 3.975 & 0.397 & 0.086 \\
\hline 1.50 & 1551 & 1058 & 0.982 & 3.929 & 0.395 & 0.072 \\
\hline 1.75 & 1556 & 1065 & 0.994 & 3.878 & 0.392 & 0.061 \\
\hline 2.00 & 1560 & 1073 & 1.017 & 3.829 & 0.390 & 0.054 \\
\hline $\mathbf{R}$ & $\begin{array}{c}\alpha / \mathbf{f} 2 \\
\mathbf{x} 10-15 \\
\text { Npm-1s2 }\end{array}$ & $\begin{array}{c}\text { Vf } \\
\text { m3mol-1 }\end{array}$ & $\begin{array}{l}\text { CE x105 } \\
\text { kJmol-1 }\end{array}$ & $\begin{array}{c}\tau \\
\times 10-12 \\
S\end{array}$ & $\begin{array}{c}\text { Za } \\
\text { x106 } \\
\text { kgm-2 s2 }\end{array}$ & Sn \\
\hline \multicolumn{7}{|c|}{ Amantadine + PEG } \\
\hline 1.473 & 1.012 & 0.011 & 1.121 & 5.135 & 1.546 & - \\
\hline 3.211 & 1.006 & 0.036 & 0.984 & 5.101 & 1.608 & 558 \\
\hline 4.990 & 1.001 & 0.071 & 0.913 & 5.075 & 1.633 & 524 \\
\hline 6.787 & 0.998 & 0.113 & 0.868 & 5.061 & 1.654 & 544 \\
\hline 8.554 & 0.992 & 0.160 & 0.835 & 5.034 & 1.678 & 586 \\
\hline 10.332 & 0.993 & 0.211 & 0.811 & 5.034 & 1.698 & 628 \\
\hline 12.104 & 1.075 & 0.236 & 0.823 & 5.450 & 1.715 & 659 \\
\hline 13.812 & 1.086 & 0.282 & 0.810 & 5.506 & 1.738 & 705 \\
\hline 15.507 & 1.086 & 0.334 & 0.796 & 5.506 & 1.760 & 747 \\
\hline \multicolumn{7}{|c|}{ Oseltamivir + PEG } \\
\hline 1.475 & 1.003 & 0.011 & 0.101 & 5.088 & 1.535 & - \\
\hline 3.277 & 1.008 & 0.038 & 0.132 & 5.113 & 1.558 & 316 \\
\hline 5.122 & 1.011 & 0.074 & 0.152 & 5.127 & 1.575 & 318 \\
\hline 6.979 & 1.008 & 0.118 & 0.168 & 5.112 & 1.593 & 358 \\
\hline 8.848 & 1.003 & 0.168 & 0.182 & 5.085 & 1.609 & 401 \\
\hline 10.695 & 1.000 & 0.222 & 0.194 & 5.070 & 1.628 & 448 \\
\hline 12.545 & 0.992 & 0.283 & 0.205 & 5.033 & 1.646 & 498 \\
\hline 14.383 & 0.985 & 0.349 & 0.215 & 4.998 & 1.664 & 542 \\
\hline 16.193 & 0.983 & 0.416 & 0.223 & 4.983 & 1.682 & 589 \\
\hline
\end{tabular}

Amantadine + Maltodextrin 


\begin{tabular}{|c|c|c|c|c|c|c|}
\hline 2.211 & 1.012 & 0.021 & 1.049 & 5.135 & 1.546 & - \\
\hline 4.236 & 1.016 & 0.055 & 0.943 & 5.152 & 1.557 & 456 \\
\hline 6.240 & 1.021 & 0.097 & 0.888 & 5.180 & 1.569 & 393 \\
\hline 8.224 & 1.032 & 0.145 & 0.853 & 5.233 & 1.582 & 395 \\
\hline 10.170 & 1.039 & 0.196 & 0.827 & 5.269 & 1.598 & 428 \\
\hline 12.101 & 1.049 & 0.250 & 0.809 & 5.318 & 1.613 & 461 \\
\hline 14.010 & 1.101 & 0.288 & 0.810 & 5.583 & 1.628 & 495 \\
\hline 15.897 & 1.125 & 0.335 & 0.803 & 5.705 & 1.643 & 531 \\
\hline 17.762 & 1.141 & 0.385 & 0.795 & 5.788 & 1.658 & 566 \\
\hline \multicolumn{7}{|c|}{ Oseltamivir + Maltodextrin } \\
\hline 2.214 & 1.003 & 0.011 & 1.113 & 5.088 & 1.535 & - \\
\hline 4.183 & 0.990 & 0.039 & 0.977 & 5.019 & 1.571 & 507 \\
\hline 6.155 & 0.982 & 0.075 & 0.910 & 4.980 & 1.590 & 463 \\
\hline 8.109 & 0.980 & 0.120 & 0.865 & 4.972 & 1.607 & 480 \\
\hline 10.022 & 0.978 & 0.169 & 0.834 & 4.962 & 1.626 & 515 \\
\hline 11.906 & 0.972 & 0.223 & 0.809 & 4.930 & 1.645 & 556 \\
\hline 13.776 & 0.971 & 0.280 & 0.790 & 4.923 & 1.663 & 595 \\
\hline 15.625 & 0.967 & 0.342 & 0.774 & 4.905 & 1.682 & 640 \\
\hline 17.480 & 0.962 & 0.399 & 0.764 & 4.882 & 1.697 & 673 \\
\hline \multicolumn{7}{|c|}{ Amantadine + SLS } \\
\hline 1.480 & 1.012 & 0.011 & 1.121 & 5.135 & 1.546 & - \\
\hline 3.337 & 1.014 & 0.038 & 0.981 & 5.145 & 1.576 & 445 \\
\hline 5.211 & 1.018 & 0.074 & 0.914 & 5.165 & 1.591 & 406 \\
\hline 7.076 & 1.020 & 0.117 & 0.871 & 5.174 & 1.607 & 425 \\
\hline 8.902 & 1.022 & 0.164 & 0.840 & 5.185 & 1.627 & 462 \\
\hline 10.739 & 1.023 & 0.215 & 0.816 & 5.191 & 1.645 & 502 \\
\hline 12.558 & 1.048 & 0.261 & 0.806 & 5.316 & 1.662 & 538 \\
\hline 14.401 & 1.071 & 0.309 & 0.797 & 5.433 & 1.674 & 565 \\
\hline 16.188 & 1.127 & 0.340 & 0.803 & 5.715 & 1.690 & 598 \\
\hline \multicolumn{7}{|c|}{ Oseltamivir + SLS } \\
\hline 1.482 & 1.003 & 0.011 & 1.113 & 5.088 & 1.535 & - \\
\hline 3.347 & 1.009 & 0.039 & 0.977 & 5.119 & 1.566 & 389 \\
\hline 5.227 & 1.014 & 0.075 & 0.910 & 5.141 & 1.581 & 363 \\
\hline 7.097 & 1.010 & 0.120 & 0.865 & 5.123 & 1.597 & 386 \\
\hline 8.973 & 1.013 & 0.169 & 0.834 & 5.137 & 1.610 & 412 \\
\hline 10.831 & 1.012 & 0.223 & 0.809 & 5.135 & 1.625 & 445 \\
\hline 12.678 & 1.014 & 0.280 & 0.790 & 5.143 & 1.640 & 480 \\
\hline 14.514 & 1.013 & 0.342 & 0.774 & 5.138 & 1.657 & 520 \\
\hline 16.317 & 1.024 & 0.399 & 0.764 & 5.191 & 1.673 & 559 \\
\hline
\end{tabular}

In all three systems, velocity gradually increases with the concentration of drugs. The variation in velocity is not much appreciable at low concentration when compared to a higher concentration. Velocity is also found to be high in Amantadine + PEG system than the other two systems. This behavior may be explained as follows the solvent molecules are highly associated, which may cause a strong association between themselves, leading to a high state of incompressibility, hence the maximum velocity and minimum association for the weak association of the molecules.

When a drug is added to aqueous PEG, Maltodextrin, and SLS solution, eventual modification may be produced due to the drug-induced ionic interaction. The water surrounding the drug is polarized by the ionic field [22], which lowers the free energy of water. When drug molecules interact with PEG, Maltodextrin, and SLS segment approach one another, the amount of intervening water decreases.

The ultrasonic velocity, density, and viscosity increase with the increase of concentration at $303 \mathrm{~K}$. However, adiabatic compressibility and intermolecular free length decrease with the increase of concentration [23,24]. The solute molecule's motion is affected by mutual-interactions between macromolecule and solvent molecule and interactions between one macromolecule with another. Indirectly, the first type of mutual interaction is termed as hydrodynamic screening, which is significant in determining the viscous flow of dilute PEG, Maltodextrin, and SLS solution. In more concentrated solutions, direct segment-segment 
interaction will exist [25]. The interaction rises to associate with polymer,dextrin, and drug molecules, which may be responsible for the increase in ultrasonic velocity.

The variation of velocity is high in Amantadine (1\%) + PEG compared with the other two systems. It may be due to the higher number of available solvated molecules for the interaction in this system. The rapid decrease of adiabatic compressibility with the increase of drug concentration in this system clearly indicates the formation of more tightly bound systems. This could be caused by the more rigid liquid structure associated with PEG's hydrogen bonding, Maltodextrin, with the drug in water. The number of available sites for hydrogen bonding is quite high in PEG and Maltodextrin systems because of the conformation adopted by water in the presence of binary solute molecules. Such reduction in compressibility has been found in cellulose derivatives solutions, which are attributed to the changes in the compressibility of solvent molecules concerned in solutions [26].

At higher concentrations, molecules are not closed, and thus, the intermolecular free length is high. The molecules come closer in lower concentrations, and segment-segment interaction will exist, thereby decreasing the intermolecular free length [27]. The increase in the internal pressure with the increase in PEG, Maltodextrin, and SLS concentration may be due to the cohesion between solvent and solute molecules and reduced hydrodynamic volume [28]. The decrease in internal pressure with an increase in concentration is due to ions moving away from others, reducing the possibility of interaction during high concentration. Internal pressure is a measure of cohesive energy of the system; hence these variations may be due to the structure making nature of the solute in the solvent. It implies that there is a weak solutesolvent and solute-solute interaction takes place in the observed systems [29]. The increasing trend of Rao's constant with concentration indicates the availability of more number of components in a given region; this leads to the tight packing of the medium. This specific interaction indicates the solute-solute and solute-solvent type [30, 31]. The absorption coefficient increases with an increase in concentration. It indicates that the extent of complexation increases and decreases with the increase in concentration, indicating the extent of complexation decreases [32].

Free volume is the effective volume in which the center of the molecule can move when all other molecules are held fixed at their mean positions. The increase in free volume indicates the increase in the magnitude of the interaction between the drug (1\%) with PEG, Maltodextrin, and SLS. Acoustic impedance is the sound wave's impedance through the mixtures of components; increasing trend in those parameters suggests the strengthening of interaction among the components. The interaction may be solute-solute (or) solute-solvent (or) solventsolvent type [33, 34]. Sn's positive value indicates the structure forming tendency of the carbohydrate and polymer with drug higher concentrations. The negative value of $\mathrm{Sn}$ indicates the structure breaking tendency of the Maltodextrin and PEG with drugs. The Sn's resultant value depends upon solvent-solute and solute-solute interactions occurring in the solution [35]. An increase in $\mathrm{Sn}$ with concentration indicates predominant solvent-solute interaction. Thus, dipole-dipole interaction of the opposite type profoundly favors the solvating tendency.

\subsection{FTIR spectroscopic studies.}

The characteristic peak of anti-influenza viral drugs with PEG, Maltodextrin, and SLS excipients was shown in Figures 2 and 3. The characteristic frequency $v(\mathrm{OH})$ bond of Amantadine is $3341 \mathrm{~cm}^{-1}$ and in the mixture of Amantadine $+P E G$ is $3381 \mathrm{~cm}^{-1}$, but in the case of Amantadine + Maltodextrin is $3339 \mathrm{~cm}^{-1}$ and Amantadine $+\mathrm{SLS}$ is $3340 \mathrm{~cm}^{-1}$ are displayed 
in figures $2(a, b, c$ and $d)$. The characteristic frequency of strong $\mathrm{C}-\mathrm{H}$ stretching vibration was found in Amantadine + PEG in $2882 \mathrm{~cm}^{-1}$ and in Amantadine + Maltodextrin and Amantadine + SLS at $2921 \mathrm{~cm}^{-1}$ and $2854 \mathrm{~cm}^{-1}$. The peak at $1602 \mathrm{~cm}^{-1}$ indicates $\mathrm{NH}_{2}$ asymmetric bending, the peak at $1643 \mathrm{~cm}^{-1}$ and $1600 \mathrm{~cm}^{-1}$ for Amantadine + Maltodextrin and Amantadine + SLS.

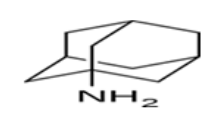

(i)

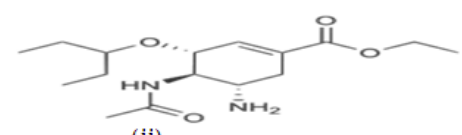

(ii)

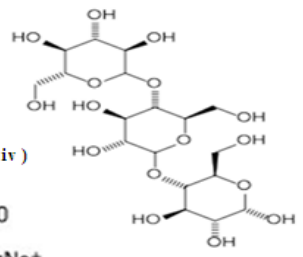

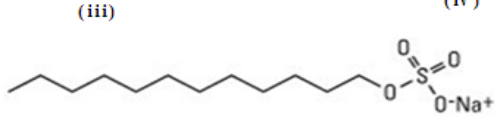

(v)

Figure 1. The Chemical Structure of (i) Amantadine (ii) Oseltamivir (iii) Polyethylene glycol (PEG) (iv) Maltodextrin (MD) (v) Sodium Lauryl Sulphate (SLS).

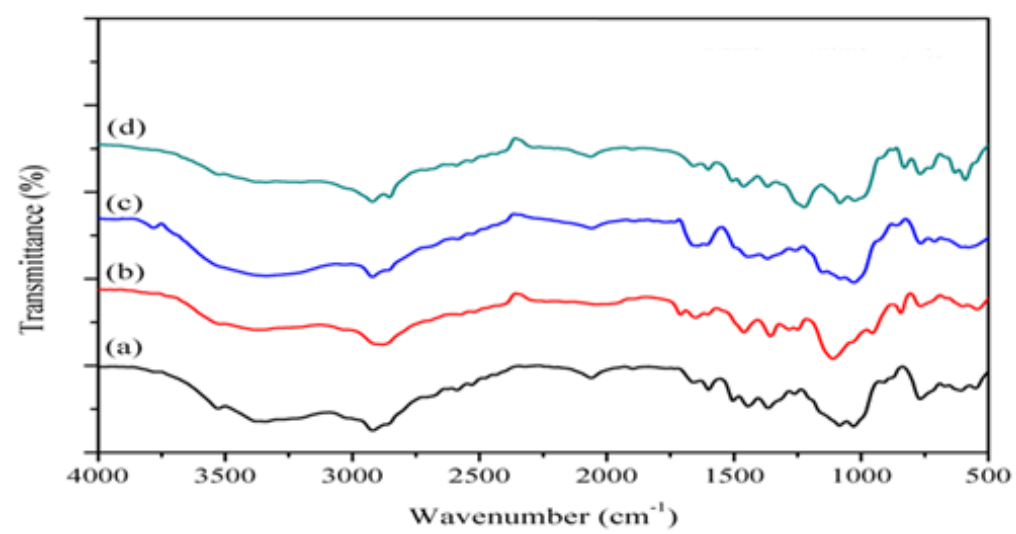

Figure 2. FTIR spectra for a) Amantadine, b) Amantadine + PEG, c) Amantadine + Maltodextrin and d) Amantadine + SLS .

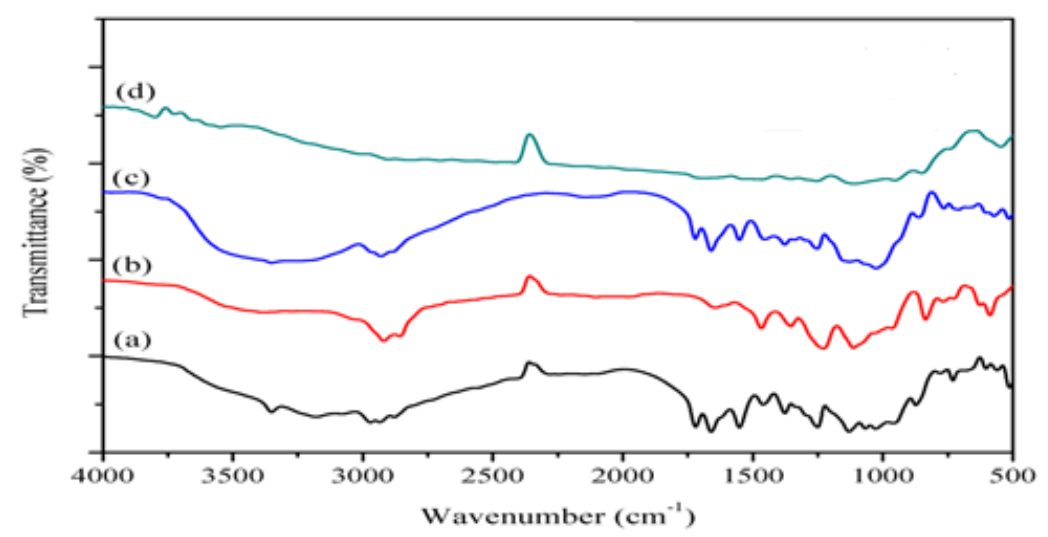

Figure 3. FTIR spectra for a) Oseltamivir, b) Oseltamivir + PEG, c) Oseltamivir + Maltodextrin and d) Oseltamivir + SLS.

The IR spectrum figure 3 (a, b, c, and d) shows that the characteristic frequency of $v(\mathrm{OH})$ bond in Oseltamivir is $3351 \mathrm{~cm}^{-1}$ and is shifted to $3382 \mathrm{~cm}^{-1}$ in Oseltamivir + PEG, 3350 $\mathrm{cm}^{-1}$ Oseltamivir + Maltodextrin, $3546 \mathrm{~cm}^{-1}$ in Oseltamivir + SLS. The observed medium level band of Oseltamivir at $2878 \mathrm{~cm}^{-1}$ of C-H stretching was shifted to $2857 \mathrm{~cm}^{-1}$ Oseltamivir + PEG, $2930 \mathrm{~cm}^{-1}$ Oseltamivir + Maltodextrin, $2902 \mathrm{~cm}^{-1}$ in Oseltamivir + SLS. In the outer 
coordination sphere of water, the hydrogen bonds are weakened due to the interaction with the anti-influenza viral drugs + Maltodextrin than PEG and SLS. Therefore this region probably corresponds to the band at the lowest wavenumber. This method is more impartial and will help diminish errors of interpretation that a bound in the highly subjective style of a visual examination of inhomogeneity arising from differences in drug mode of preparation.

\section{Conclusions}

From the above results, it can be extrapolated to the pharmacological activities in the complexation of model high - dose, poorly compressible, and fast - release anti-influenza viral drugs (Amantadine and Oseltamivir); Polymers (PEG), Dextrin (Maltodextrin), Surfactant (Sodium Lauryl Sulfate) and the interaction order was as follows in ultrasonic measurement: i) Amantadine + Maltodextrin > Amantadine + SLS > Amantadine + PEG; ii) Oseltamivir + SLS $>$ Oseltamivir + Maltodextrin > Oseltamivir + PEG

This study is an essential tool to prove the pharmaceutical drugs' selectivity, simplicity, and suitability.

\section{Funding}

This research received no external funding.

\section{Acknowledgments}

The authors express their deep sense of gratitude to S.Mullainathan Department of Physics, Thiru. Vi. Ka. Government Arts College, Thiruvarur, Tamilnadu, India, for his constant encouragement and valuable suggestions throughout this work.

\section{Conflicts of Interest}

The authors declare no conflict of interest.

\section{References}

1. Erden, N.; Çelebi, N. A study of the inclusion complex of naproxen with $\beta$-cyclodextrin. International Journal of Pharmaceutics 1988, 48, 83-89, https://doi.org/10.1016/0378-5173(88)90250-5.

2. Mura, P.; Faucci, M.T.; Bettinetti, G.P. The influence of polyvinylpyrrolidone on naproxen complexation

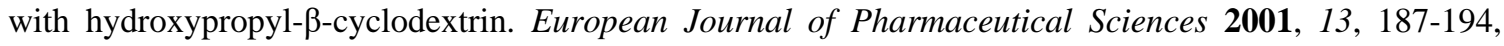
https://doi.org/10.1016/S0928-0987(01)00093-8.

3. Partyka, M.; Au, B.H.; Evans, C.H. Cyclodextrins as phototoxicity inhibitors in drug formulations: studies on model systems involving naproxen and $\beta$-cyclodextrin. Journal of Photochemistry and Photobiology A: Chemistry 2001, 140, 67-74, https://doi.org/10.1016/S1010-6030(01)00386-0.

4. Loftsson, T.J.P. Increasing the cyclodextrin complexation of drugs and drug biovailability. $1998,53$.

5. Loftsson, T.; Brewster, M.J.P.T.E. Cyclodextrins as pharmaceutical excipients. 1997, 9, 26-35.

6. Harris, J.M.Poly (ethylene glycol) chemistry: Biotechnical and Biomedical applications. Plenum, New york, 1992.

7. Chapke, U.; Berad, B.; Agrawal, P.; Meshram, B.J.J.o.G.B. Ultrasonic velocity and acoustical parameters of hybrid drugs of ambroxol hydrochloride at 300.15 K.J. Global Sci.2015, 4, 1530-1542.

8. Sunanda,A.S.; Shashikant, A.R.A.; Rajesh. H.S. Use of a new nanosized photocatelyst BaO3TiO.SrO3TiOAn ecofriendly process for removal of coloring agents, Int. J. Res. Chem. Environ.2013, 3, 89-93.

9. Illiger, S.R.; Fadnis, C.; Demappa, T. Miscibility studies of HPMC/PEG blends in water by viscosity, density, refractive index and ultrasonic velocity method, Carbohydrate polymers 2009, 75, 484-488.

10. Endo, I.; Nagamune, T.; Katoh, S.; Yonemoto, T.Bioseparation Engineering, 1st Edition, Elsevier, 2000.

11. Lawrence. R.Engineering procee for Bioseparations. Butterworth, HeinmannnWobum 1994.

12. Soane. D.S.Polymer applications in Biotechnology. Prentice Hall, Englewood cliffs 1992. 
13. Valero, M.; Carrillo, C. Effect of binary and ternary polyethyleneglycol and/or $\beta$-cyclodextrin complexes on the photochemical and photosensitizing properties of Naproxen. Journal of Photochemistry and Photobiology B: Biology 2004, 74, 151-160, https://doi.org/10.1016/j.jphotobiol.2004.03.004.

14. Valero, M.; Esteban, B. Effect of binary and ternary polyvinylpyrrolidone and/or hydroxypropyl- $\beta$ cyclodextrin complexes on the photochemical and photosensitizing properties of Naproxen. Journal of $\begin{array}{llllll}\text { Photochemistry and Photobiology } & \text { B: } & \text { Biology } & \text { 2004, } & \text { 76, }\end{array}$ 102,https://doi.org/10.1016/j.jphotobiol.2004.06.005.

15. Uekama, K.; Hirayama, F.; Irie, T. Cyclodextrin Drug Carrier Systems. Chemical Reviews 1998, 98, $2045-$ 2076, https://doi.org/10.1021/cr970025p.

16. Sweetman, S.C.Martindale. The Complete Reference. 33th ed., Pharmaceutical Press, London, $2002 ; \mathrm{pp}$. 635-636.

17. Loftsson, T.; Fridriksdottir, H.; Sigurdardottir, A.M.; Ueda, H. The effect of water soluble polymers on drug cyclodextrin complexation, Int. J. Pharm.1994, 110, 169-177.

18. Loftsson, T.; Fridriksdottir, H.; Sigurdardottir, A.M. The effect of polymers on cyclodextrin complexation“. In Proceedings of the 7th International Cyclodextrins Symposium, (T. Osa et al., Eds.), Business Center for Academic societies Japan, Tokyo, 1994; pp. 218 - 221.

19. Ganzerli, G.; Van Santvliet, L.; Verschuren, E.; Ludwig, A.J.P. Influence of beta-cyclodextrin and various polysaccharides on the solubility of fluorescein and on the rheological and mucoadhesive properties of ophthalmic solutions. Pharmazie 1996, 51, 357-362.

20. Nithiyanantham, S.; Palaniappan, L. Ultrasonic investigation on aqueous polysaccharide (starch) at 298.15 K. Arabian Journal of Chemistry 2014, 7, 272-276, https://doi.org/10.1016/j.arabjc.2010.10.030.

21. Vanathi, V.; Mullainathan, S.; Nithiyanatham, S.; Ramasamy, V.; Palaniappan, L. Ultrasonic velocity, density, viscosity for the ternary mixture of (benzene + chloroform + cyclohexane) at different temperatures. Heliyon 2019, 5, https://doi.org/10.1016/j.heliyon.2019.e02203.

22. Punitha, S.; Uvarani, R.; Panneerselvam, A.; Nithiyanantham, S. Physico-chemical studies on binary aqueous solutions of Anti-Viral Influenza drugs. Heliyon 2019, 5, https://doi.org/10.1016/j.heliyon.2019.e01941.

23. Nithiyanantham, S.; Palaniappan, L. Physico-Chemial and Excess Thermo Acoustical Study on Some Monosaccharide (Galactose) with Enzyme Amylase in Aqueous Media at 298.15 K. Journal of Bionanoscience 2013, 7, 292-295, https://doi.org/10.1166/jbns.2013.1127.

24. Nithiyanantham, S.; Palaniappan, L. Thermodynamic Studies of Lactose with Amylase in Aqueous Media at 298.15 K. Journal of Computational and Theoretical Nanoscience 2012, 9, 2193-2197, https://doi.org/10.1166/jctn.2012.2638.

25. Dzida, M.; Zorębski, E.; Zorębski, M.; Żarska, M.; Geppert-Rybczyńska, M.; Chorążewski, M.; Jacquemin, J.; Cibulka, I. Speed of Sound and Ultrasound Absorption in Ionic Liquids. Chemical Reviews 2017, 117, 3883-3929, https://doi.org/10.1021/acs.chemrev.5b00733.

26. Punitha, S.; Uvarani, R; Panneerselvam, A; Effect of $\mathrm{pH}$ is aqueous Hydroxy Propyl Methyl Cellulose) polymer solution. Results in Materials 2020, 7, 100120.

27. Nithiyanantham, S.; Palaniappan, L. Ultrasonic studies on aqueous monosaccharides with enzyme amylase. Journal of Molecular Liquids 2016, 221, 401-407, https://doi.org/10.1016/j.molliq.2016.05.069.

28. Nithiyanantham, S.; Palaniappan, L. Ultrasonic study of adsorption in disaccharide (maltose) metabolism. Applied Acoustics 2010, 71, 754-758, https://doi.org/10.1016/j.apacoust.2010.03.007.

29. Nithiyanantham, S.; Palaniappan, L.Thermodynamical and Thermoacoustical study on some monosaccharides (glucose) with enzyme amylase in aqueous media at 298.15 K., Euro. Phy. J. Appl. Phys. 2011, 3 .

30. Kannappan, V.; Ali, S.; Mahaboob, P. Determination of stability constants of charge transfer complexes of iodine monochloride and certain ethers in solution at $303 \mathrm{~K}$ by ultrasonic method. Ind. J. Pure and Appl. Phys.2006, 44, 903-908.

31. Nithiyanantham, S.; Palaniappan, L. Acoustical and Physico-chemical study of binary azeotropes (aniline), J. Mol. Liqds. 2020, 113423, https://doi.org/10.1016/j.molliq.2020.113423.

32. Nithiyanantham, S.; Mullainathan, S.; Jeyalakshmi, R.Thermo acoustical studies on some electrolytic solvent mixtures at $303 \mathrm{~K}$, Oriental J Chem.2009, 25, 759-760.

33. Punitha, S.; Uvarani, R.; Panneerselvam, R.; Nithiyanantham, S.Physico Chemical studies on some saccharides in aqueous cellulose solutions at different temperature - Acoustical and FTIR analysis. J Saudi chem. Soc.2014, 18, 657-665.

34. Prakash, B.; Suresh, S.; Narendra, C.B.J.A.P. Physicochemical characterization of $\beta$-cyclodextrin and hydroxy ethyl $\beta$-cyclodextrin complexes of rifampicina.Ars Pharm.2006, 47, 37-59. 